Journal for ImmunoTherapy of Cancer

\title{
G-CSF as a suitable alternative to GM- CSF to boost dinutuximab-mediated neutrophil cytotoxicity in neuroblastoma treatment
}

Paula Martinez Sanz (10 , ${ }^{1}$ Dieke J van Rees, ${ }^{1}$ Lieke M J van Zogchel, ${ }^{2,3}$ Bart Klein, ${ }^{1}$ Panagiota Bouti, ${ }^{1}$ Hugo Olsman, ${ }^{4}$ Karin Schornagel, ${ }^{1}$ Ivana Kok, ${ }^{1}$ Ali Sunak, ${ }^{1}$ Kira Leeuwenburg, ${ }^{1}$ Ilse Timmerman, ${ }^{2,5}$ Miranda P Dierselhuis, ${ }^{2}$ Waleed M Kholosy, ${ }^{2}$ Jan J Molenaar, ${ }^{2}$ Robin van Bruggen, ${ }^{1}$ Timo K van den Berg, ${ }^{4,6}$ Taco W Kuijpers, ${ }^{1,7}$ Hanke L Matlung, ${ }^{1}$ Godelieve A M Tytgat, ${ }^{2}$ Katka Franke ${ }^{1}$

To cite: Martinez Sanz P, van Rees DJ, van Zogchel LMJ, et al. G-CSF as a suitable alternative to GM-CSF to boost dinutuximab-mediated neutrophil cytotoxicity in neuroblastoma treatment. Journal for ImmunoTherapy of Cancer 2021;9:e002259. doi:10.1136/jitc-2020-002259

- Additional supplemental material is published online only. To view, please visit the journal online (http://dx.doi.org/10. 1136/jitc-2020-002259).

PMS, DJvR, HLM, GAMT and KF contributed equally.

Accepted 26 March 2021

Check for updates

(C) Author(s) (or their employer(s)) 2021. Re-use permitted under CC BY-NC. No commercial re-use. See rights and permissions. Published by BMJ.

For numbered affiliations see end of article.

Correspondence to

Paula Martinez Sanz;

p.martinezsanz@sanquin.nl

\section{ABSTRACT}

Background Current immunotherapy for patients with high-risk neuroblastoma involves the therapeutic antibody dinutuximab that targets GD2, a ganglioside expressed on the majority of neuroblastoma tumors. Opsonized tumor cells are killed through antibody-dependent cellular cytotoxicity (ADCC), a process mediated by various immune cells, including neutrophils. The capacity of neutrophils to kill dinutuximab-opsonized tumor cells can be further enhanced by granulocyte-macrophage colonystimulating factor (GM-CSF), which has been shown in the past to improve responses to anti-GD2 immunotherapy. However, access to GM-CSF (sargramostim) is limited outside of Northern America, creating a high clinical need for an alternative method to stimulate dinutuximab responsiveness in the treatment of neuroblastoma. In this in vitro study, we have investigated whether clinically wellestablished granulocyte colony-stimulating factor (G-CSF) can be a potentially suitable alternative for GM-CSF in the dinutuximab immunotherapy regimen of patients with neuroblastoma.

Methods We compared the capacity of neutrophils stimulated either in vitro or in vivo with GM-CSF or G-CSF to kill dinutuximab-opsonized GD2-positive neuroblastoma cell lines and primary patient tumor material. Blocking experiments with antibodies inhibiting either respective Fc gamma receptors ( $\mathrm{Fc} \gamma \mathrm{R})$ or neutrophil integrin $\mathrm{CD} 11 \mathrm{~b} /$ CD18 demonstrated the involvement of these receptors in the process of ADCC. Flow cytometry and live cell microscopy were used to quantify and visualize neutrophilneuroblastoma interactions.

Results We found that G-CSF was as potent as GMCSF in enhancing the killing capacity of neutrophils towards neuroblastoma cells. This was observed with in vitro stimulated neutrophils, and with in vivo stimulated neutrophils from both patients with neuroblastoma and healthy donors. Enhanced killing due to GM-CSF or G-CSF stimulation was consistent regardless of dinutuximab concentration, tumor-to-neutrophil ratio and concentration of the stimulating cytokine. Both GM-CSF and G-CSF stimulated neutrophils required Fc $\gamma R$ Rlla and CD11b/CD18 integrin to perform $A D C C$, and this was accompanied by trogocytosis of tumor material by neutrophils and tumor cell death in both stimulation conditions.

Conclusions Our preclinical data support the use of G-CSF as an alternative stimulating cytokine to GMCSF in the treatment of high-risk neuroblastoma with dinutuximab, warranting further testing of G-CSF in a clinical setting.

\section{INTRODUCTION}

Neuroblastoma, a tumor originating from the early embryonic neural crest, is the most common extracranial solid tumor diagnosed in children. The median age at diagnosis is 19 months and it accounts for almost $15 \%$ of all cancer-related mortality in children. ${ }^{1}$ The prognosis and treatment options for this neuroendocrine tumor, generally arising in the adrenal glands and sympathetic ganglia, are determined by the stage of the disease. For very low-risk, low-risk and intermediaterisk categories-as classified by the International Neuroblastoma Risk Group, which uses molecular, pathological as well as clinical criteria for patient classification ${ }^{2}$ - the risk of recurrence is minimal. However, the prognosis for high-risk neuroblastoma remains poor despite intensive multimodal treatment comprising surgery, chemotherapy, myeloablative therapy with stem cell rescue and radiotherapy. ${ }^{34}$ In 2015 , the Food and Drug Administration approved the addition of the therapeutic antibody dinutuximab to the maintenance phase of the treatment protocol for patients with highrisk neuroblastoma (online supplemental figure 1). This combination of dinutuximab with the existing multimodal treatment increased the survival rate from high-risk neuroblastoma to $50 \% .^{5}$ Nonetheless, still 
half of the patients relapse and succumb to the tumor. Increasing the efficacy of dinutuximab is therefore of utmost importance.

The chimeric monoclonal antibody dinutuximab binds GD2, a ganglioside present on the surface of neuroblastoma cells. Upon binding, dinutuximab marks the cells for immune-mediated destruction via antibody-dependent cellular cytotoxicity (ADCC) by Fc gamma receptor (Fc $\gamma R$ ) expressing immune cells. ${ }^{6}{ }^{7}$ Natural killer (NK) cells and macrophages play a prominent role in mediating ADCC in diverse cancer types. In neuroblastoma, however, neutrophils have been described as the major cell population involved in dinutuximab-mediated killing of neuroblastoma cells in vitro. ${ }^{8}$ The cytotoxic activity of dinutuximab can be significantly enhanced when given in combination with cytokines that specifically stimulate the activity of immune cells. Particularly, granulocytemacrophage colony-stimulating factor (GM-CSF), stimulating neutrophils and macrophages, and interleukin-2 (IL-2), stimulating NK cells, were demonstrated to positively contribute to the efficacy preclinically ${ }^{8-10}$ and also in clinical trials. ${ }^{11} 12$

Notably, a partially randomized phase III trial showed a survival benefit for patients with high-risk neuroblastoma treated during alternating cycles of dinutuximab combined with GM-CSF or IL-2, and isotretinoin, as compared with patients treated with isotretinoin alone (COG ANBL0032 study). ${ }^{12}$ This trial led to the standardization of this combination regimen for the maintenance phase in the treatment protocol for patients with high-risk neuroblastoma in the USA (online supplemental figure 1) ${ }^{513}$ However, the addition of IL-2 has later been shown to bring minimal clinical improvement for dinutuximabtreated patients with neuroblastoma, ${ }^{14} 15$ and access to GM-CSF for clinical use outside of Northern America is limited. ${ }^{16}$ Therefore, the immunotherapy regimen for patients with high-risk neuroblastoma in Europe is not fully defined with regard to the cytokine administration, resulting in a potentially increased risk of suboptimal treatment.

In this study, we aim at providing preclinical evidence for the use of an alternative stimulating cytokine if GM-CSF is unavailable, to ultimately increase dinutuximab responsiveness in the treatment protocol of neuroblastoma. Based on the known ability of granulocyte colony-stimulating factor (G-CSF) to enhance neutrophilmediated ADCC, ${ }^{17-20}$ we propose the use of this clinically well-established, and broadly available cytokine. Furthermore, we investigated the potentially negative effect of G-CSF on neuroblastoma cells as an additional safety measure, since several studies suggested G-CSF treatment of chemotherapy-induced neutropenia to cause alterations in tumor cell phenotype, promoting neuroblastoma tumorigenicity. ${ }^{21-23}$ Our preclinical data on G-CSF efficacy in neutrophil activity against neuroblastoma support the clinical use of G-CSF as it potentiates neutrophil-mediated ADCC of dinutuximab-opsonized neuroblastoma cells to the same extent as GM-CSF.
MATERIALS AND METHODS

\section{Patients, controls and samples}

For in vivo GM-CSF stimulated neutrophils, remnant heparinized blood was used from patients with high-risk neuroblastoma at the Princess Máxima Center during the different GM-CSF cycles of the dinutuximab treatment protocol (specified in online supplemental table 1 and online supplemental figure 1), for which Biobank approval was obtained. These patients received GM-CSF as part of the immunotherapy regimen, which was given as maintenance therapy after induction and consolidation phases of the treatment protocol, according to the COG ANBL0032 study. Here, GM-CSF $\left(250 \mu \mathrm{g} / \mathrm{m}^{2} /\right.$ day, sargramostim, Leukine) was administered subcutaneously in course 1, 3 and 5 for 14 days. After the first three consecutive GM-CSF doses, the blood was sampled prior to the fourth dose of GM-CSF and before dinutuximab treatment started. As control, healthy unrelated donor neutrophils were used.

For in vivo G-CSF stimulated neutrophils, heparinized blood was collected at Sanquin from granulocyte transfusion donors $\sim 30$ hour after subcutaneous injection of $10 \mu \mathrm{g} / \mathrm{kg}$ clinical grade G-CSF (filgrastim, Neupogen). As control, heparinized blood was collected from healthy unrelated volunteers, as well as at least 3 weeks later (when G-CSF is cleared from circulation) ${ }^{24-27}$ from the same G-CSF injected healthy donor.

\section{Neutrophil isolation and in vitro stimulation}

Heparinized peripheral blood was diluted 1:2 with phosphate-buffered saline (PBS) $+10 \%$ trisodium citrate and separated by density gradient centrifugation over isotonic Percoll $(1.076 \mathrm{~g} / \mathrm{mL}$, GE Healthcare). The pellet fraction, containing both erythrocytes and granulocytes, underwent erythrocyte lysis with ice cold hypotonic ammonium chloride solution $\left(155 \mathrm{mM} \mathrm{NH} \mathrm{Nl}_{4}, 10 \mathrm{mM}\right.$ $\mathrm{KHCO}_{3}, 0.1 \mathrm{mM}$ EDTA in water). After isolation, $5 \times 10^{6}$ / $\mathrm{mL}$ neutrophils were resuspended in 4-(2-hydroxyethyl) -1-piperazineethanesulfonic acid supplemented with 5 $\mathrm{g} / \mathrm{L}$ human albumin (Albuman, Sanquin Plasma Products), $1 \mathrm{mM} \mathrm{CaCl}$ and $5.5 \mathrm{mM}$ glucose. ${ }^{28}$

Neutrophils were either used directly after isolation (referred to as unstimulated neutrophils) or were stimulated overnight at $37^{\circ} \mathrm{C}$ and $5 \% \mathrm{CO}_{2}$ with either $10 \mathrm{ng} / \mathrm{mL}$ recombinant human GM-CSF (Peprotech) or $10 \mathrm{ng} / \mathrm{mL}$ clinical grade G-CSF (Neupogen), unless otherwise specified. After overnight incubation, the percentage of apoptotic cells was determined using Annexin V staining (BD Biosciences) to correct for the number of viable neutrophils (the percentage of apoptotic neutrophils typically ranged between $10 \%$ and $30 \%$ ) prior to any experiments.

\section{Cell culture}

The human neuroblastoma cell lines NMB, IMR-32 and LAN-1 were obtained from the Leibniz Institute, Germany. These cell lines were routinely cultured at $37^{\circ} \mathrm{C}$ and $5 \% \mathrm{CO}_{2}$ and maintained in Iscove's Modified Dulbecco's Medium (IMDM, Gibco) supplemented with $20 \%$ of 
heat-inactivated fetal calf serum (FCS), $2 \mathrm{mM}$ L-glutamine, 100 units $/ \mathrm{mL}$ penicillin and $100 \mu \mathrm{g} / \mathrm{mL}$ streptomycin (further referred to as IMDM complete medium) for a maximum of 30 passages. NMB cells were harvested using trypsin; IMR-32 and LAN-1 cells were harvested by tapping the culture flask and resuspending the culture medium. The human neuroblastoma cell lines SHEP-2, SK-N-AS, SH-SY5Y and SK-N-BE (kindly provided by the Department of Oncogenomics, Amsterdam UMC) were routinely cultured at $37^{\circ} \mathrm{C}$ and $5 \% \mathrm{CO}_{2}$ and maintained in Dulbecco's Modified Eagle Medium (DMEM, Gibco) completed with $20 \%$ of FCS, $0.1 \mathrm{mM}$ non-essential amino acids, $2 \mathrm{mM}$ L-glutamine, 100 units $/ \mathrm{mL}$ penicillin and $100 \mu \mathrm{g} / \mathrm{mL}$ streptomycin for maximum of 30 passages. These cells grew adherent as well as in suspension and were harvested by collecting supernatant as well as by using trypsin.

\section{Primary patient-derived neuroblastoma cells}

The primary patient-derived neuroblastoma spheroid line AMC691B (further referred to as 691B) was derived from a bone marrow metastasis (B) of patient $691 .{ }^{29} 691 \mathrm{~B}$ cells grow in spheroids and were cultured and maintained in DMEM with low glucose and sodium pyruvate (Invitrogen) supplemented with 25\% Ham's F12 nutrient mixture (Invitrogen), $1 \times$ B-27 supplement minus vitamin A (50×, Gibco), $1 \times$ N-2 supplement (100×, Gibco), $20 \mathrm{ng} /$ $\mathrm{mL}$ animal-free human epidermal growth factor (Peprotech), $40 \mathrm{ng} / \mathrm{mL}$ human basic fibroblast growth factor (Peprotech), $200 \mathrm{ng} / \mathrm{mL}$ human insulin-like growth factor (Peprotech), $10 \mathrm{ng} / \mathrm{mL}$ human platelet-derived growth factor (PDGF)-AA (Peprotech), $10 \mathrm{ng} / \mathrm{mL}$ human PDGF-BB (Peprotech), 100 units/mL penicillin and 100 $\mu \mathrm{g} / \mathrm{mL}$ streptomycin for maximum of 24 passages. To obtain a single-cell suspension, cells were treated with Accutase solution for 5 min (Sigma-Aldrich).

\section{Chromium-based ADCC assay}

Target cells $\left(1 \times 10^{6}\right)$ were labeled with $100 \mu \mathrm{Ci}{ }^{51} \mathrm{Cr}$ (PerkinElmer) for $90 \mathrm{~min}$ at $37^{\circ} \mathrm{C}$ and washed with PBS. Chromium-labeled target cells $\left(5 \times 10^{3}\right)$ were co-incubated with neutrophils in a 96-well U-bottom plate (Corning) in the absence or presence of dinutuximab (Unituxin, Ch14.18, United Therapeutics) in the appropriate culture medium for 4 hours at $37^{\circ} \mathrm{C}$ and $5 \% \mathrm{CO}_{2}$. A target:effector (T:E) ratio of 1:50 (ie, 5000:250,000 cells) and a final concentration of $0.5 \mathrm{\mu g} / \mathrm{mL}$ of dinutuximab were used, unless specified otherwise. Spontaneous and maximum ${ }^{51} \mathrm{Cr}$ release were determined by incubating the target cells without effector cells and by treating the target cells with a $0.1 \%$ Triton X-100 solution in culture medium, respectively. After incubation, $30 \mu \mathrm{L}$ of supernatant was subsequently transferred to Lumaplates (PerkinElmer). The plates were dried overnight at room temperature and analyzed in a MicroBeta ${ }^{2}$ plate reader (PerkinElmer). The percentage of cytotoxicity was calculated as: [ (experimental counts per minute (CPM))-spontaneous CPM)/ (maximum CPM-spontaneous CPM) ]×100\%. All conditions were performed in triplicate.

For Fc $\gamma$ receptor blocking experiments, $F\left(a^{\prime}\right)_{2}$ fragments against Fc $\gamma$ RIIa (CD32, clone 7.3, Ancell) or FcyRIIIb (CD16, clone 3G8, Ancell) were used and compared with isotype control mIgG1 F(ab') ${ }_{2}$ fragments (clone MOPC 31C, Ancell). Using purified human IgG Fc fragments (Bethyl, USA), we aimed to saturate the highaffinity Fc $\gamma$ RI. Blocking reagents were pre-incubated with neutrophils at $10 \mu \mathrm{g} / \mathrm{mL}$ for $45 \mathrm{~min}$ at room temperature. Subsequently, the effector cells were used in the chromium-based ADCC assay.

For integrin blocking experiments, $\mathrm{F}\left(\mathrm{ab}{ }^{\prime}\right)_{2}$ fragments against CD18 (clone IB4, Ancell) were pre-incubated with neutrophils at $10 \mu \mathrm{g} / \mathrm{mL}$ for $15 \mathrm{~min}$ at room temperature, after which the cells were used in the chromium-based ADCC assay.

\section{Trogocytosis assay}

The trogocytosis of neuroblastoma cells by neutrophils was quantified using flow cytometry and measured by the uptake of tumor cell membrane by the neutrophils. Tumor cells were stained with $2 \mu \mathrm{M}$ lipophilic membrane dye 3,3'-dioctadecyloxacarbocyanine perchlorate ( $\mathrm{DiO}$, Invitrogen); neutrophils were labeled with $0.625 \mathrm{ng}$ Calcein Violet-AM (Invitrogen) for $30 \mathrm{~min}$ at $37^{\circ} \mathrm{C}$. After labeling, populations were washed twice with PBS. Cells were co-incubated at a T:E ratio of 1:5 (ie, 50,000:250,000 cells) in the absence or presence of $0.5 \mu \mathrm{g} / \mathrm{mL}$ dinutuximab in a U-bottom 96-well plate (Greiner Bio-One) for $60 \mathrm{~min}$ at $37^{\circ} \mathrm{C}$ and $5 \% \mathrm{CO}_{2}$ in IMDM complete medium. After incubation, cells were fixed with STOPbuffer (PBS containing $20 \mathrm{mM}$ sodium fluoride, $0.5 \%$ paraformaldehyde (PFA) and $1 \%$ bovine serum albumin) and analyzed using flow cytometer Canto II (BD Biosciences). The neutrophil population (all Calcein Violet- $\mathrm{AM}^{+}$events) was assessed for the mean fluorescence intensity of membrane dye DiO. Data were analyzed with FlowJo software (V.10.6.1, Becton Dickinson, Ashland, Oregon, USA).

\section{Live cell imaging}

NMB target cells labeled with $5 \mu \mathrm{M}$ lipophilic membrane dye 1,1'-dioctadecyl-3,3,3',3'-tetramethylindodicarbocyanine, 4-chlorobenzenesulfonate salt (DiD, Invitrogen) and $2.5 \mathrm{nM}$ cytoplasmic dye Calcein Red-Orange-AM (ThermoFisher) were co-incubated with unstained neutrophils at a T:E ratio of 1:5 in glass chambered coverslips (Ibidi) of $9.4 \times 10.7 \times 6.8 \mathrm{~mm}^{3}$ well dimensions. Two drops of the DNA-binding Nuc-Green dye were added in the extracellular medium before imaging. Cells were co-incubated for periods up to 4 hours at $37^{\circ} \mathrm{C}$ and $5 \%$ $\mathrm{CO}_{2}$ in the presence of $0.5 \mu \mathrm{g} / \mathrm{mL}$ dinutuximab in IMDM complete medium. Imaging was performed within 5 min after co-incubation of tumor cells and neutrophils and lasted up to $210 \mathrm{~min}$ using a Leica TCM SP8 confocal microscope (Leica). 


\section{Flow cytometry staining}

For neutrophil characterization, cells were stained with $10 \mu \mathrm{g} / \mathrm{mL}$ fluorescein isothiocyanate (FITC)-labeled antibodies: anti-Fc $\gamma \mathrm{RI}$ (CD64, clone 10.1, Bio-Rad), anti-Fc $\gamma$ RII (CD32, clone AT10, Bio-Rad), anti-Fc $\gamma$ RIII (CD16, clone 3G8, BD Biosciences), anti-CD11b (clone CLB-mon-gran/1, B2, Sanquin Reagents) and anti-CD18 (clone MEM48, Diaclone).

For target cell characterization, human anti-GD2 antibody dinutuximab (Unituxin, Ch14.18, United Therapeutics) was used to quantify GD2 expression by titrating dinutuximab from $10 \mu \mathrm{g} / \mathrm{mL}$ to $0.001 \mu \mathrm{g} / \mathrm{mL}$. Secondary antibody Alexa Fluor 647 goat antihuman $\operatorname{IgG}$ F(ab') fragment (Jackson ImmunoResearch) was used for detection. To determine expression of G-CSF receptor on neuroblastoma cells, $20 \mu \mathrm{g} / \mathrm{mL}$ of anti-CD114 PE-Cyanine7 (BD Biosciences) was used. Cell viability of tumor cells was determined using Hoechst 33342 solution (Invitrogen). All incubations took place for $20 \mathrm{~min}$ on ice in the dark. The appropriately labeled IgG isotypes were used to correct for any potential background. After washing, cells were resuspended in PBS supplemented with $6 \mathrm{~g} / \mathrm{L}$ human albumin (Albuman, Sanquin Plasma Products) and fluorescence was measured on a Canto II flow cytometer (BD Biosciences). Data were analyzed with FlowJo software (V.10.6.1).

\section{Effect of G-CSF on JAK/STAT3 pathway}

Tumor cell samples were exposed to $10 \mathrm{ng} / \mathrm{mL}$ G-CSF (Neupogen) for 0, 5, 10 and $20 \mathrm{~min}$. Hereafter, cells were fixed with $4 \%$ PFA, permeabilized with ice-cold $90 \%$ methanol and stained with fluorescently labeled antibodies for total STAT3-PerCp-Cyanine5.5 (BD Biosciences) and phospho-STAT3-PE (pSTAT3, BD Biosciences) as previously described. ${ }^{30}$ Neutrophils were used as control in this setting. Fluorescence was measured on a Canto II flow cytometer (BD Biosciences) and data were analyzed with FlowJo software (V.10.6.1).

\section{Effect of G-CSF on neuroblastoma cell proliferation rates}

Tumor cells were cultured in the presence or absence of $10 \mathrm{ng} / \mathrm{mL}$ clinical grade G-CSF (Neupogen) in the appropriate culture medium for $1-3$ weeks. The medium supplemented with cytokine was refreshed twice a week where applicable; $0.3 \times 10^{6}$ IMR-32 cells or $0.5 \times 10^{6} 691 \mathrm{~B}$ cells were plated in each well of a 6 -well plate (Corning) and the proliferation rate of these cultures was determined by counting the cells using a CASY Cell Counter (Roche Innovatis). The population doubling time of G-CSF-treated cultures was calculated with a doubling time calculator (http://www.doubling-time.com/ compute.php).

\section{RNA isolation, cDNA synthesis and RT-quatitative PCR}

Total RNA was extracted from neuroblastoma cell lines on days $0,7,14$ and 21 after G-CSF exposure by using the QIAamp RNA Blood Mini Kit (Qiagen) according to manufacturer's instructions. Complementary DNA
(cDNA) was synthesized from 2 to $3 \mu \mathrm{g}$ RNA, using the High-Capacity RNA-to-cDNA Kit (Applied Biosystems), as described previously. ${ }^{31}$ Quatitative PCR for reference gene beta-glucoronidase (GUSB), adrenergic neuroblastoma markers ${ }^{32} 33$ paired-like homeobox 2b (PHOX2B), cholinergic receptor nicotinic alpha 3 (CHRNA3), dopamine beta hydroxylase $(D B H)$ and tyrosine hydroxylase (TH), and mesenchymal neuroblastoma markers ${ }^{34}$ paired related homeobox 1 (PRRX1) and periostin (POSTN) was performed using the Viia7 (Applied Biosystems) as previously described. ${ }^{31}$ Normalization for expression was based on the expression of GUSB with the equation: normalized threshold cycle $(\mathrm{dCt})=\left(\mathrm{Ct}_{\text {marker }}-\mathrm{Ct}_{\mathrm{GUSB}}\right)$. All reactions were performed in triplicate (except GUSB, which was performed in duplicate) and mean values were used. As a positive control, a calibration curve of neuroblastoma cell line IMR-32 was used for the adrenergic markers, plasmids were used for GUSB and the mesenchymal panel to establish the PCR efficiency.

\section{Statistical analysis}

Differences between groups were assessed using GraphPad Prism 8. Specific test and number of individual biological replicates (n) are indicated in figure legends for each experiment. When $p$ values were $\leq 0.05$, differences were deemed significant; error bars indicate the SEM.

\section{RESULTS \\ GM-CSF and G-CSF equally enhance neutrophil-mediated ADCC of neuroblastoma cells}

To compare the effect of GM-CSF with G-CSF on the tumor cell killing capacity of neutrophils, we performed ADCC experiments with various GD2-positive and GD2negative neuroblastoma cells (all cell line characteristics are summarized in online supplemental table 1, titration of dinutuximab depicted in online supplemental figure 2A-C) using neutrophils that were unstimulated or stimulated overnight with the respective cytokines in vitro. Unstimulated neutrophils were not able to kill dinutuximab-opsonized GD2-positive neuroblastoma cell lines NMB, IMR-32 and LAN-1, whereas stimulation of neutrophils either with GM-CSF or G-CSF enhanced the cytotoxicity levels significantly, with neither cytokine being superior to the other (figure 1A). The effect of either GM-CSF or G-CSF stimulation on the killing efficacy remained equal, irrespective of the concentration of dinutuximab tested (figure $1 \mathrm{~B}$ and online supplemental figure $3 \mathrm{~A}$ ), different $\mathrm{T}: \mathrm{E}$ ratios (figure $1 \mathrm{C}$ and online supplemental figure $3 \mathrm{~B}$ ) or the various concentrations of GM-CSF and G-CSF used to stimulate neutrophils (figure 1D and online supplemental figure 3C). The high concentrations of dinutuximab (figure 1B, exceeding $5 \mu \mathrm{g} / \mathrm{mL}$ ) led to decreased cytotoxicity, probably due to the formation of immune complexes. We observed no killing of GD2-negative neuroblastoma cell lines SH-SY5Y and SK-N-BE in the presence of dinutuximab, regardless of the stimulating cytokine used (online supplemental 
A $\square$ Unstimulated $\square$ GM-CSF in vitro $\square$ G-CSF in vitro D

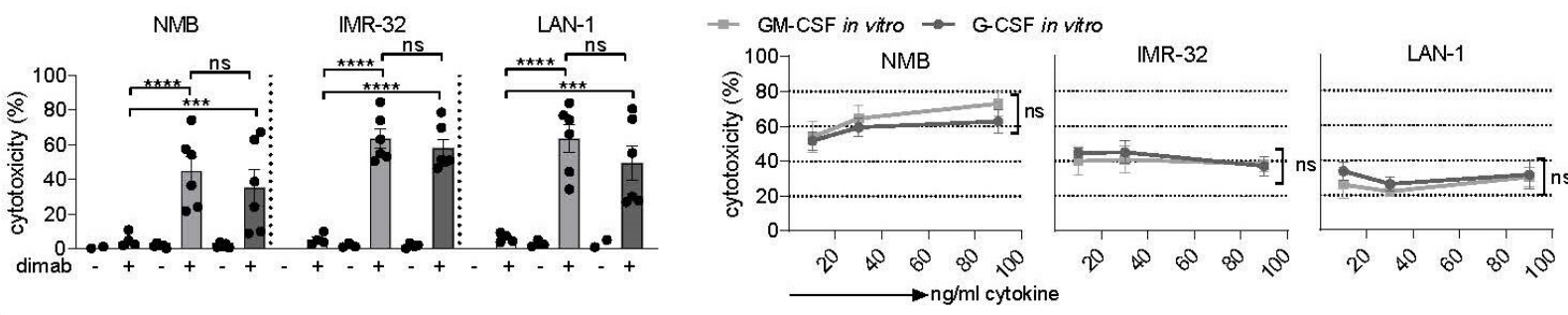

B

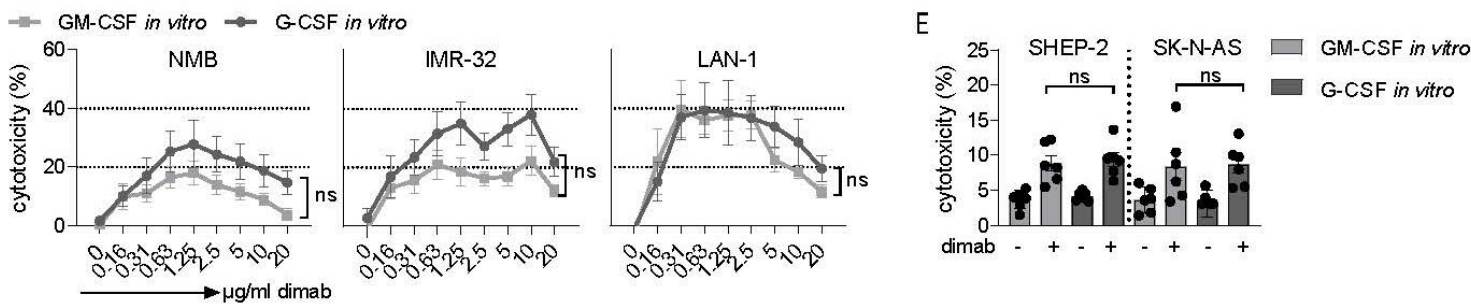

$\mathrm{C}$
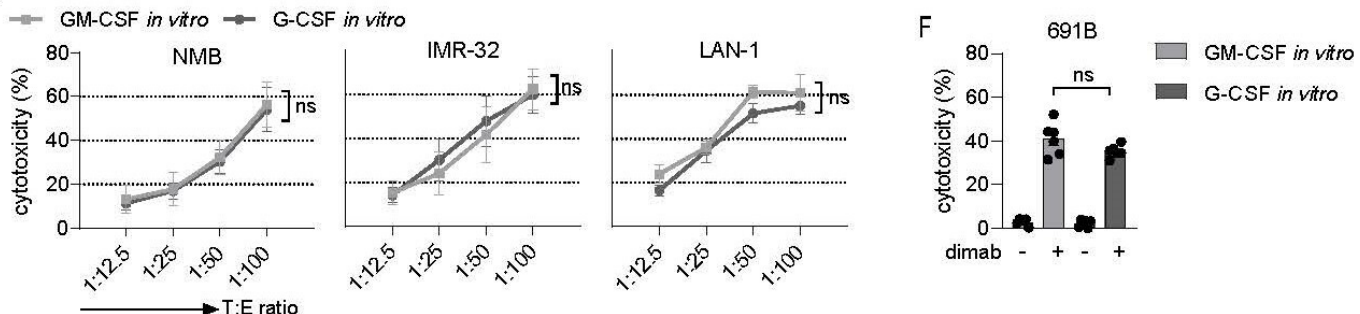

Figure 1 GM-CSF and G-CSF equally enhance neutrophil-mediated ADCC of neuroblastoma cells. (A) ADCC of NMB, IMR-32 and LAN-1 cells opsonized with $(+)$ or without $(-)$ dinutuximab (dimab) by unstimulated neutrophils (white bars) or stimulated in vitro with GM-CSF (light gray bars) or G-CSF (dark gray bars). NMB $n=6$, IMR-32 $n=4-6$, LAN-1 $n=4-6$ of three individual experiments. Statistical significance was tested with an ordinary one-way ANOVA with post hoc Sidak test. (B) ADCC of NMB, IMR-32 and LAN-1 cells opsonized with increasing concentrations of dinutuximab, from 0 to $20 \mu \mathrm{g} / \mathrm{mL}$, by neutrophils stimulated in vitro with GM-CSF (light gray squares) or G-CSF (dark gray circles). NMB $n=4$, IMR-32 $n=6$, LAN-1 $n=4$ of three individual experiments. Statistical differences were assessed with an unpaired T-test on AUC; online supplemental figure 3A). (C) ADCC of dinutuximab-opsonized NMB, IMR-32 and LAN-1 cells by neutrophils stimulated in vitro with GM-CSF (light gray squares) or G-CSF (dark gray circles) at different T:E ratios ranging from 1:12.5 to 1:100. NMB $n=4$, IMR-32 $n=4$, LAN-1 $n=6$ of three individual experiments. Statistical differences were tested with an unpaired T-test on AUC (online supplemental figure 3B). (D) ADCC of dinutuximab-opsonized NMB, IMR-32 and LAN-1 cells by neutrophils stimulated in vitro with increasing concentrations of GM-CSF (light gray squares) or G-CSF (dark gray circles), from 10 to $90 \mathrm{ng} / \mathrm{mL}$. NMB $n=4$, IMR-32 $\mathrm{n}=4$, LAN$1 \mathrm{n}=4$ of two individual experiments. Statistical significance was assessed with an unpaired T-test on AUC (online supplemental figure 3C). (E) ADCC of SHEP-2 and SK-N-AS cells opsonized with (+) or without (-) dinutuximab (dimab) by neutrophils stimulated in vitro with GM-CSF (light gray bars) or G-CSF (dark gray bars). SHEP-2 $n=6$, SK-N-AS $n=6$ of three individual experiments. Statistical differences were assessed with an unpaired T-test. (F) ADCC of primary patient-derived 691B cells opsonized with (+) or without (-) dinutuximab (dimab) by neutrophils stimulated in vitro with GM-CSF (light gray bars) or GCSF (dark gray bars). N=6 of three individual experiments. Statistical significance was tested with an unpaired T-test. ADCC, antibody-dependent cellular cytotoxicity; ANOVA, analysis of variance; AUC, areas under curve; G-CSF, granulocyte colonystimulating factor; GM-CSF, granulocyte-macrophage colony-stimulating factor; ns, not significant. ${ }^{* \star *} p<0.001 ;{ }^{* \star \star \star} p<0.0001$.

figure 2D). Neuroblastoma cell lines SHEP-2 and SK-NAS, expressing lower levels of GD2 (online supplemental figure 2B), were also killed by stimulated neutrophils irrespective of the cytokine used (figure 1E), although at lower levels than the GD2-positive cell lines NMB, IMR-32 and LAN-1 (figure 1A). To test the ability of both cytokines to promote neutrophil-mediated killing of primary patient material, we used GM-CSF and G-CSF stimulated neutrophils in an ADCC assay with GD2 expressing 691B cells derived from bone marrow metastasis of a patient with high-risk neuroblastoma (characteristics in online supplemental figure 2C and online supplemental table 1). Both stimuli induced effective killing of primary tumor cells and no differences were seen between the two cytokines (figure 1F). Together, these observations show that the in vitro stimulating effect of G-CSF is as effective as GM-CSF in boosting neutrophil-mediated ADCG of neuroblastoma cells.

\section{GM-CSF and G-CSF both mediate neutrophil ADCC through Fc $\gamma R$ Rlla and CD11b/CD18 integrins}

In order to perform ADCC, neutrophils need expression of Fc $\gamma$ receptors (neutrophils can express Fc $\gamma$ RI, Fc $\gamma$ RIIa/c and Fc $\gamma$ RIIIb) and CD11b/CD18 integrin. ${ }^{35} 36$ As stimulation with GM-CSF or G-CSF enhances tumor cell killing (figure 1), we explored whether the killing of 
A
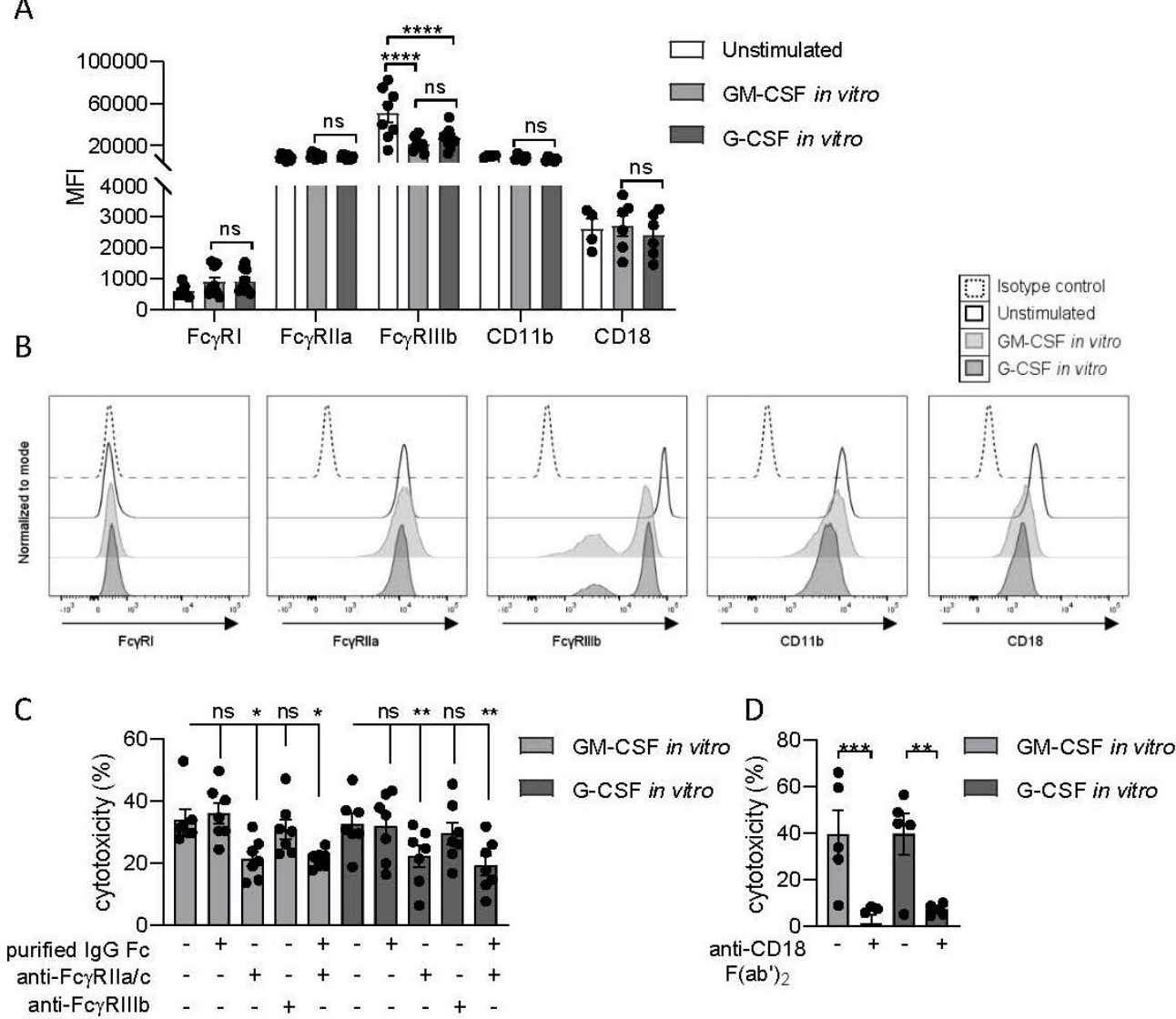

Figure 2 GM-CSF andG-CSF both mediate neutrophil ADCC through FcyRlla and CD11b/CD18 integrins. (A) Fc $\gamma$ receptor and CD11b/CD18 integrin expression (expressed as MFI) on neutrophils after in vitro GM-CSF (light gray bars) or G-CSF (dark

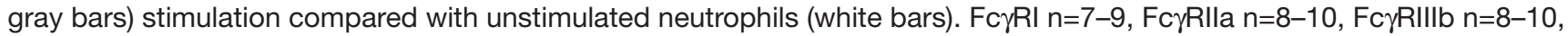
CD11b $n=4-6, C D 18 n=4-6$ of five individual experiments. Statistical differences were tested with ordinary one-way ANOVA with post hoc Dunnett test. (B) Representative histogram of flow cytometry analysis of (from left to right) FcyRl, FcyRlla, FcyRlllb, CD11b and CD18 expression on unstimulated neutrophils (in white), and neutrophils stimulated in vitro with GM-CSF (in light gray) or G-CSF (in dark gray). The dashed line depicts an isotype control. (C) ADCC of dinutuximab-opsonized NMB cells by in vitro stimulated neutrophils with GM-CSF (light gray bars) or G-CSF (dark gray bars). Fcy receptors are blocked or

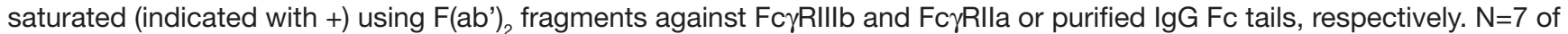
four individual experiments. Statistical significance was tested with ordinary one-way ANOVA with post hoc Sidak test. (D) ADCC of dinutuximab-opsonized NMB cells by in vitro stimulated with GM-CSF (light gray bars) or G-CSF (dark gray bars) without (-) or with (+) CD18 integrin block with $\mathrm{F}\left(\mathrm{ab} \mathrm{b}^{\prime}\right)_{2}$ fragments. $\mathrm{N}=5$ of three individual experiments. Statistical differences with ordinary one-way ANOVA with post hoc Sidak test. ADCC, antibody-dependent cellular cytotoxicity; ANOVA, analysis of variance; G-CSF, granulocyte colony-stimulating factor; GM-CSF, granulocyte-macrophage colony-stimulating factor; MFI, mean fluorescence intensity; ns, not significant. ${ }^{*} p<0.05 ;{ }^{* *} p<0.01 ;{ }^{* * *} p<0.001 ;{ }^{* * * *} p<0.0001$.

tumor cells by differently stimulated neutrophils required presence of these molecules in a similar fashion. Freshly isolated, unstimulated, neutrophils constitutively express low-affinity and intermediate-affinity Fc $\gamma$ RIIa or Fc $\gamma R I I c$ $(\mathrm{CD} 32 \mathrm{a} / \mathrm{c})$ and FcyRIIIb (CD16b), respectively. It has been shown that on stimulation with G-CSF neutrophils upregulate the expression of FcyRI (CD64) and shed Fc $\gamma$ RIIIb. ${ }^{18} 3738$ We showed that overnight stimulation of neutrophils with either GM-CSF or G-CSF in vitro significantly lowered Fc $\gamma$ RIIIb expression compared with unstimulated neutrophils, whereas Fc $\gamma$ RIIa expression remained unaltered on stimulation. Fc $\gamma$ RI expression increased slightly but this was not statistically significant. No differences in neutrophil Fc $\gamma$ receptor levels were observed between GM-CSF and G-CSF stimulation (figure 2A,B). Next to this, we investigated which Fc $\gamma$ receptor(s) neutrophils need to facilitate killing of neuroblastoma cells. Previous studies showed that Fc $\gamma$ RIIa is the major Fc $\gamma$ receptor that mediates the killing of antibodyopsonized solid cancer cells. ${ }^{35} 36{ }^{39}$ Indeed, blocking Fc $\gamma$ RIIa using $\mathrm{F}(\mathrm{ab})$, fragments reduced ADCC of dinutuximab-opsonized NMB cells for both GM-CSF and G-CSF overnight stimulated neutrophils, while blocking

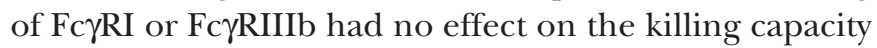
of neutrophils, regardless of the stimulating cytokine used (figure 2C).

In addition to $\mathrm{Fc} \gamma$ receptors, neutrophils need functional expression of the heterodimer integrin $\mathrm{CD} 11 \mathrm{~b} /$ CD18 to perform ADCC..$^{35} 38$ After overnight in vitro 
stimulation with either GM-CSF or G-CSF, the expression of $\mathrm{CD} 11 \mathrm{~b}$ and $\mathrm{CD} 18$ remained similar to unstimulated neutrophils (figure 2A,B). When blocking CD18 using $\mathrm{F}(\mathrm{ab}$ ') , fragments, known to inhibit $\mathrm{CD} 11 \mathrm{~b} / \mathrm{CD} 18$ integrin function, ${ }^{35}$ tumor cell killing was abolished similarly for both GM-CSF and G-CSF stimulated neutrophils (figure 2D), suggesting that both cytokines stimulate neutrophils to kill neuroblastoma cells through functional $\mathrm{CD} 11 \mathrm{~b} / \mathrm{CD} 18$ integrins.

\section{GM-CSF and G-CSF stimulation induce trogocytosis of neuroblastoma cells by neutrophils and is accompanied by tumor cell death}

The ability of immune cells to perform trogocytosis, an active mechanism involving the uptake of plasma membrane from a donor cell, is well-known. ${ }^{41}$ In the recent years, it has become clear that trogocytosis can also be a cytotoxic mechanism, at least in the context of antibody-dependent tumor cell killing by myeloid cells. ${ }^{42}$ More specifically, neutrophil-mediated trogocytosis in which the neutrophil takes 'bites' from the plasma membrane of antibody-opsonized cancer cells can result in cancer cell lysis, also known as trogoptosis. This has been described for trastuzumab-opsonized breast cancer cells and it has been shown to be dependent on functional Fc $\gamma$ RIIa and CD11b/CD18 integrins. ${ }^{36}$ To investigate whether GM-CSF and G-CSF stimulated neutrophils could trogocytose neuroblastoma cells, we performed a FACS-based trogocytosis assay where we labeled overnight stimulated neutrophils with Calcein Violet-AM and freshly harvested NMB cells with the membrane dye DiO (figure 3A). In conditions with dinutuximab, neutrophils stimulated with either GM-CSF or G-CSF became positive for the membrane dye $\mathrm{DiO}$, indicative of trogocytosis (figure 3A,B), although this was significantly higher in G-CSF stimulated neutrophils. In an attempt to demonstrate whether trogocytic events by neutrophils coincided with tumor cell death, we performed live cell confocal imaging on dinutuximab-opsonized cells. We labeled NMB cells with membrane dye DiD and cytoplasmic dye Calcein Red-Orange-AM, which were co-incubated with stimulated neutrophils. As a live/dead indicator, a membrane-impermeable DNAbinding dye was added to the extracellular medium during imaging. During co-incubation of tumor cells with neutrophils stimulated overnight with either GM-CSF or G-CSF, we observed that neutrophils became positive for the neuroblastoma membrane dye, but not the cytoplasmic dye (which would indicate phagocytosis; figure 3C). In addition, we found that neutrophil trogocytic interactions were followed with tumor cell death as appreciated by the staining of nuclear material exposed to the extracellular medium on membrane disruption induced by the attacking neutrophils (figure 3C). Collectively, these data support a link between trogocytic events and subsequent tumor cell death.
G-CSF treatment does not alter neuroblastoma cell phenotype in vitro

Currently, G-CSF is used to treat chemotherapyinduced neutropenia, often occurring in patients with high-risk neuroblastoma, ${ }^{43}$ which was shown to be an advantageous addition to the treatment protocol. ${ }^{44}$ However, although not generally supported by clinical or in vitro data, previously published studies have suggested a possible role of G-CSF with regard to neuroblastoma proliferation and invasive properties of neuroblastoma cell lines. ${ }^{21}$ Also, the tumorigenicity and metastasis formation in human xenograft and murine neuroblastoma tumor models were suggested to be enhanced in some studies. ${ }^{22}{ }^{23}$ Neuroblastoma cells express the G-CSF receptor (figure 4A) and therefore we investigated whether exposure of neuroblastoma cells to G-CSF could alter their phenotype and possibly make them more resistant towards neutrophil-mediated killing. First, we investigated the effect of G-CSF on its cognate receptor signaling. Activation of STAT3 is known to take place downstream of the G-CSF receptor after ligand binding ${ }^{45}$ and therefore we measured the level of STAT3 phosphorylation (pSTAT3) by intracellular flow cytometry staining of IMR-32 cells that were exposed to the cytokine for 0 , 5, 10 or $20 \mathrm{~min}$. Compared with neutrophils used as positive control, IMR-32 cells did not show any phosphorylation of STAT3, suggesting no STAT3-mediated signaling through the G-CSF receptor (online supplemental figure $4 \mathrm{~A}, \mathrm{~B}$ ). To verify that no other (ie, longterm) effects due to G-CSF binding to its receptor occurred, we cultured IMR-32 and patient-derived 691B neuroblastoma cells in the absence or presence of G-CSF for up to 3 weeks, as this is the period during which clinical grade pegylated G-CSF stays in circulation. ${ }^{24-27}$ No changes on GD2 or G-CSF receptor expression were detected over time and expression remained high at all time points tested (7, 14 and 21 days; online supplemental figure 4C-F). During incubation with G-CSF, the proliferation rates of tumor cell cultures were similar to control, with no proliferative advantage of the G-CSF exposed cells (figure 4B and online supplemental figure $4 \mathrm{G}-\mathrm{J}$ ). Since IMR-32 and 691B cells have an adrenergic (epithelial) phenotype $^{34}$ (online supplemental table 1), we studied whether exposure to G-CSF initiated epithelial-tomesenchymal transition (EMT) in these cells. The transition from an adrenergic to a mesenchymal phenotype is known to increase invasive properties of tumor cells facilitating metastasis. ${ }^{46-48}$ We performed quantitative RT-PCR on RNA samples isolated from IMR-32 and 691B cells cultured with or without G-CSF. We investigated mRNA expression of several adrenergic (PHOX2B, CHRNA3, DBH and TH) and mesenchymal (PRRX1 and POSTN) markers that have been described to be specific for neuroblastoma. ${ }^{32-34}$ Overall, no differences in mRNA expression were seen for any of the mesenchymal markers PRRX1 and 
A GM-CSF in vitro

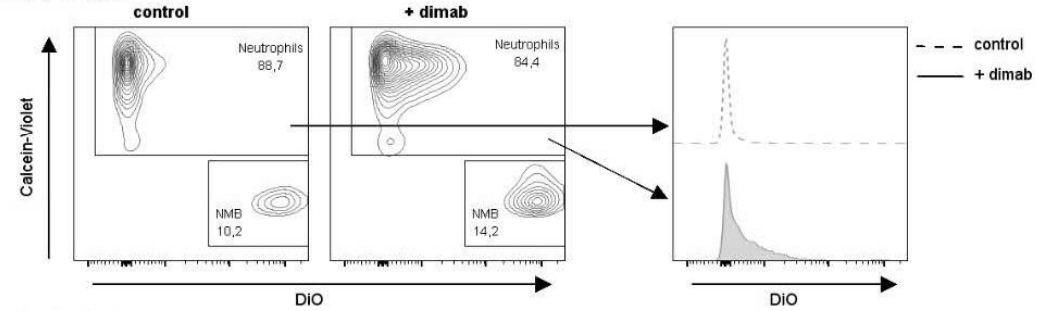

G-CSF in vitro
B

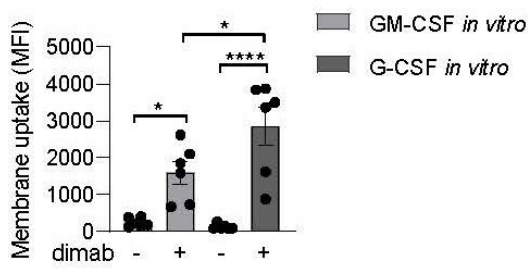

Figure 3 GM-CSF and G-CSF stimulation induce trogocytosis of neuroblastoma cells by neutrophils. (A) Representative flow cytometry plots (left) and histogram (right) of a trogocytosis experiment, where Calcein Violet-AM-labeled neutrophils are distinguished from DiO-labeled tumor cells. Note the increase for membrane dye DiO in the neutrophils in conditions with dinutuximab (+dimab in flow cytometry plots and continuous line in histogram) as compared with conditions without dinutuximab (control in flow cytometry plots and dashed line in histogram). In vitro GM-CSF stimulated neutrophils are depicted in the top panels; in vitro G-CSF stimulated neutrophils are depicted in the bottom panels. Numbers indicated are the percentages of the mentioned populations. (B) NMB neuroblastoma membrane uptake by neutrophils in vitro stimulated with GM-CSF (light gray bars) or G-CSF (dark gray bars) in the presence or absence of dinutuximab (dimab). Data depict the $\mathrm{MFI}$ of membrane dye $\mathrm{DiO}$ in the neutrophil population. $\mathrm{N}=6$ of three individual experiments. Statistical differences were tested with ordinary one-way ANOVA with post hoc Sidak test. (C) Live cell confocal imaging stills showing neutrophils (white arrows) stimulated with GM-CSF (upper panels) or G-CSF (lower panels) in vitro taking up pieces of membrane of DiD (green) and Calcein Red-Orange-AM-labeled (orange) NMB cells, opsonized with dinutuximab. Note the uptake of the membrane label DiD only, and no uptake of the orange cytoplasmic dye by neutrophils. As an indication of cell death, note how neutrophil trogocytic interactions are directly followed by tumor cell membrane permeabilization and NucGreen staining of nuclear material (blue) once exposed to the extracellular medium. Time (min) is set to 0 from the moment a neutrophil approached the tumor cell and is indicated in the upper left corner of each still. Imaging took place within 210 min from start of the recording for all movies. Scale bar represents $20 \mu \mathrm{M}$. ANOVA, analysis of variance; DiD, 1,1'-Dioctadecyl-3,3,3',3'-Tetramethylindodicarbocyanine, 4-Chlorobenzenesulfonate salt; DiO, 3,3'-dioctadecyloxacarbocyanine perchlorate; G-CSF, granulocyte colony-stimulating factor; GM-CSF, granulocyte-macrophage colony-stimulating factor; MFI, mean fluorescence intensity. ${ }^{\star} p<0.05$; ${ }^{\star \star \star *} p<0.0001$.

POSTN during G-CSF culture at any of the time points (7, 14 and 21 days) when compared with the control (untreated) condition. Also, no changes of adrenergic markers were detected, implying no signs of EMT (figure 4C,D). Last, the susceptibility of G-CSF exposed neuroblastoma cells towards neutrophil ADCC was studied by co-incubating IMR-32 cells cultured for $0,7,14$ and 21 days with G-CSF with overnight in vitro G-CSF stimulated neutrophils (figure $4 \mathrm{E}$ ). No differences were found in the extent of neutrophilmediated cytotoxicity between G-CSF cultured tumor cells and control tumor cells. Altogether, these results 
A [1 Istype control G-C-Str receptcr staring IMR-32

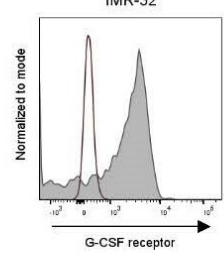

$691 \mathrm{~B}$

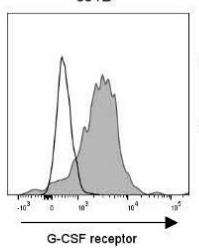

B

-a Control $\rightarrow-$ G-CSF in culture

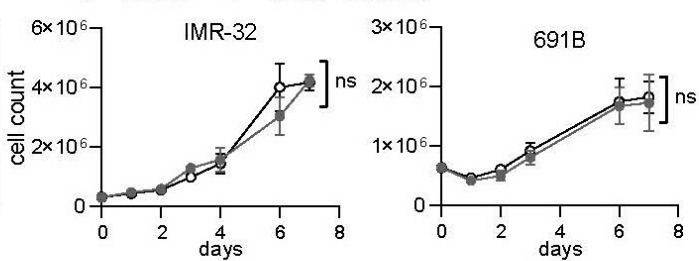

D
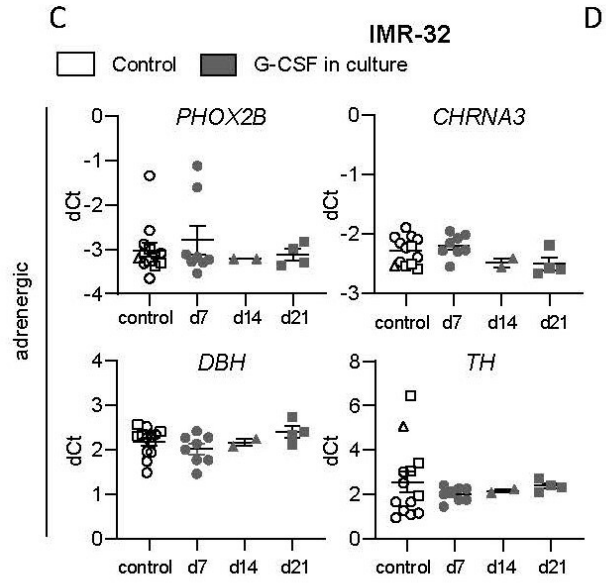

691B
$\square$ Control $\square$ G-CSF in culture
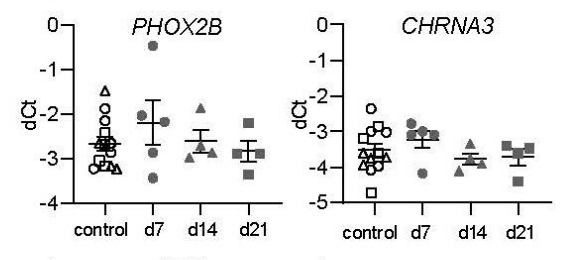

E $\square$ control $\square$ G-CSF in culture
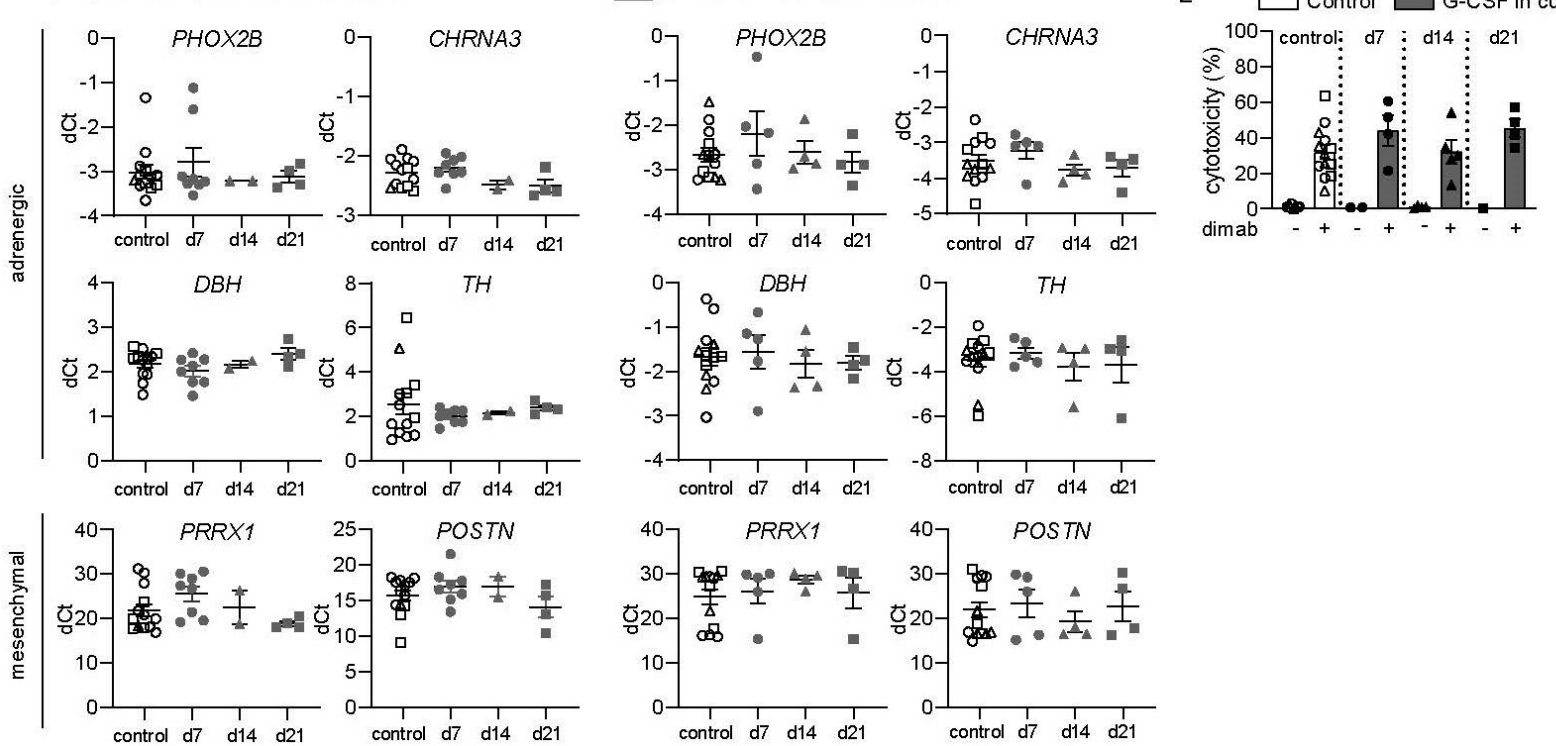

Figure 4 G-CSF treatment does not alter neuroblastoma cell phenotype. (A) Representative histograms depicting G-CSF receptor expression (gray) on IMR-32 (left panel) and 691B cells (right panel). (B) Proliferation curves of IMR-32 (left panel) and 691B (right panel) when cultured in the absence (control, white circles) or presence (gray circles) of G-CSF for 7 days. IMR-32 $n=4,691 B n=3$ of three and four individual experiments, respectively. Statistical significance was assessed with a paired T-test on AUC (online supplemental figure 3C,D). (C, D) Normalized expression (dCt, delta cycle threshold $=\mathrm{Ct}_{\text {marker }}-\mathrm{Ct}_{\text {GusB }}$ ) levels of adrenergic neuroblastoma markers PHOX2B, CHRNA3, DBH and TH, as well as mesenchymal neuroblastoma markers PRRX1 and POSTN on IMR-32 cells (C) and patient-derived 691B cells (D) cultured in the absence (control, white symbols) or presence of G-CSF for 7 (gray circles), 14 (gray triangles) or 21 (gray squares) days. IMR-32 n=2-8, 691B $n=4-5$ of two individual experiments. Statistical differences were tested with ordinary one-way ANOVA with post hoc Sidak test. (E) ADCCof IMR-32 cells cultured in the absence (control, white symbols) or presence of G-CSF (gray bars) for 7 (circles), 14 (triangles) or 21 (squares) days opsonized with (+) or without (-) dinutuximab (dimab) by in vitro G-CSF stimulated neutrophils. $\mathrm{N}=4-13$ of five individual experiments. Statistical significance was tested with ordinary one-way ANOVA with post hoc Sidak test. ADCC, antibody-dependent cellular cytotoxicity; ANOVA, analysis of variance; G-CSF, granulocyte colony-stimulating factor; AUC, areas under curve; ns, not significant.

show that in vitro G-CSF has no detectable effect on neuroblastoma phenotype, nor on the susceptibility of tumor cells towards neutrophil-mediated ADCC.

\section{Comparable ex vivo killing of neuroblastoma cells by in vivo GM-CSF or G-CSF stimulated neutrophils}

Although in vitro GM-CSF and G-CSF stimulated neutrophils show enhanced killing capacity towards neuroblastoma cells (figure 1), whether this also occurs in vivo and in patients with neuroblastoma is still unclear. To take this a step closer to the anticipated situation in patients, we investigated the respective abilities of GM-CSF and G-CSF at potentiating neutrophils to kill neuroblastoma cells after in vivo stimulation. We obtained blood from patients with high-risk neuroblastoma that were administered GM-CSF subcutaneously for three consecutive days prior to blood sampling (patient characteristics are summarized in online supplemental table 2). In addition, we collected blood from granulocyte transfusion donors $\sim 30$ hours after subcutaneous G-CSF injection. We found similar Fc $\gamma$ receptor expression profiles for both GM-CSF and G-CSF in vivo stimulated neutrophils. Compared with unstimulated neutrophils, in vivo G-CSF stimulated neutrophils, as well as in vivo GM-CSF stimulated neutrophils showed a significant increase of Fc $\gamma \mathrm{RI}$ expression and a decrease

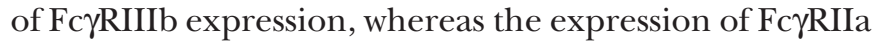
remained unaltered (figure 5A-D), similar as observed 
for the in vitro stimulated neutrophils (figure 2A,B). The capacity of in vivo stimulated neutrophils to kill dinutuximab-opsonized neuroblastoma cells was investigated ex vivo. Neutrophils from GM-CSF injected patients with neuroblastoma induced significantly greater cytotoxicity levels of the GD2-positive neuroblastoma cell lines NMB and IMR-32 as compared with unstimulated neutrophils (figure $5 \mathrm{E}$ and online supplemental figure 5). Likewise, the cytotoxic ability of in vivo G-CSF stimulated neutrophils was similarly enhanced (figure 5F). Overall, this indicates that both cytokines can stimulate neutrophils in vivo to kill neuroblastoma cells ex vivo.

\section{DISCUSSION}

High-risk neuroblastoma is an aggressive cancer affecting children mostly before the first year of age. Therapy consists of intense multimodal treatment,

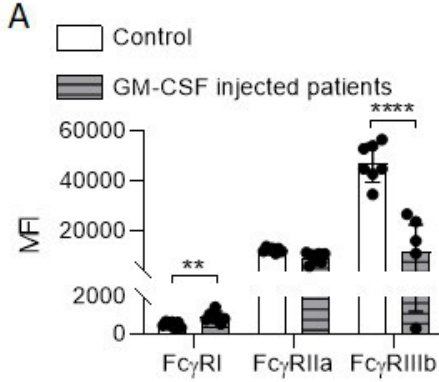

B

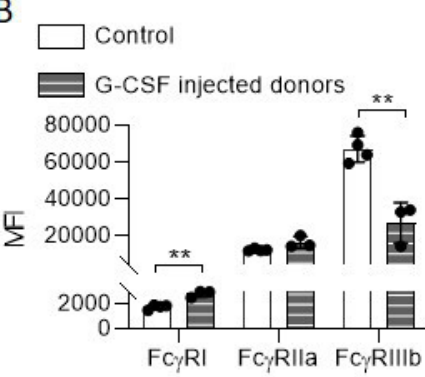

$\mathrm{E}$

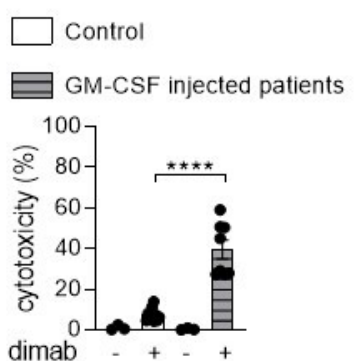

C
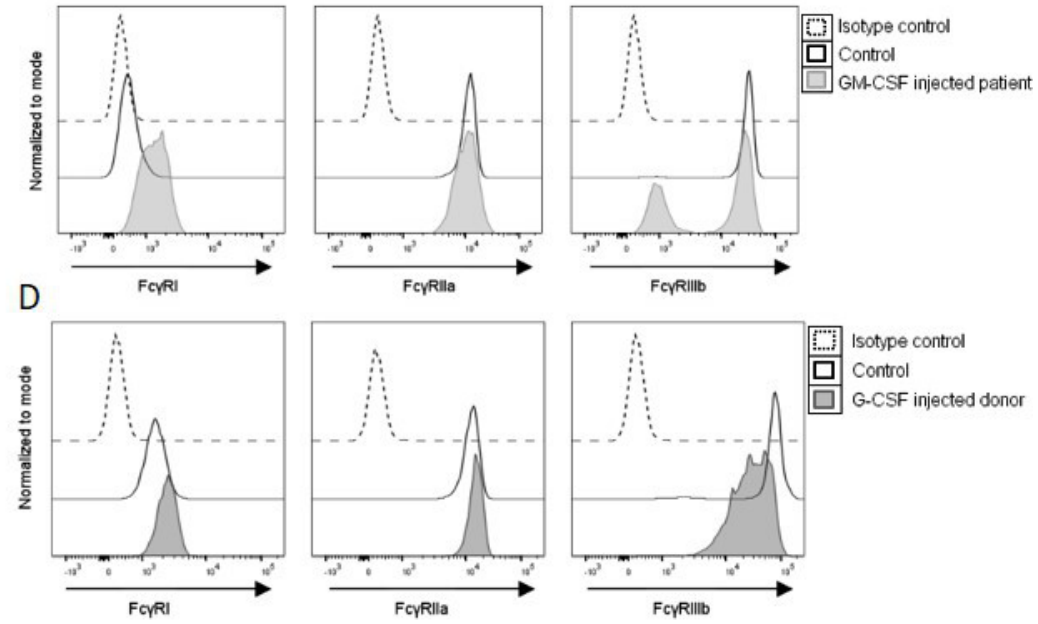

$\mathrm{F}$

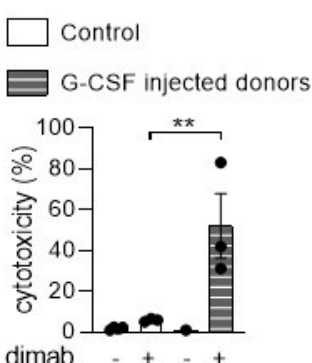

Figure 5 Comparable ex vivo killing of neuroblastoma cells by in vivo GM-CSF or G-CSF stimulated neutrophils. (A) Fcy receptor expression (expressed as MFI) on neutrophils from patients with neuroblastoma that were injected with GM-CSF (striped light gray bars) compared with control neutrophils from healthy donors (white bars). Both for healthy donors and patients, $F c \gamma R I n=7$, Fc $\gamma R$ Ila $n=7$, FcyRlllb $n=7$ of four individual experiments. Statistical analysis was assessed with an unpaired T-test. (B) Fc $\gamma$ receptor expression (expressed as MFI) on neutrophils from healthy donors that were injected with G-CSF (striped dark gray bars) compared with control neutrophils from healthy donors (white bars). Both for healthy donors and G-CSF injected

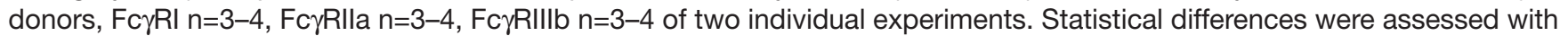

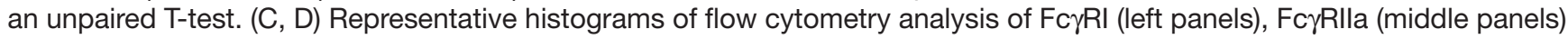
and FcyRIllb (right panels) expression on control neutrophils from healthy donors (in white) compared with neutrophils from patients with neuroblastoma that were injected with GM-CSF (in light gray) (C), or neutrophils from healthy donors that were administered G-CSF (in dark gray) (D). The dashed line depicts an isotype control. (E) Antibody-dependent cellular cytotoxicity (ADCC) of NMB cells opsonized with (+) or without (-) dinutuximab (dimab) by control neutrophils from healthy donors (white bars) or by neutrophils from patients with neuroblastoma that were administered GM-CSF (striped light gray bars). N=10 healthy donors, $n=8$ patients of five individual experiments. Statistical differences were assessed with ordinary one-way ANOVA with post hoc Sidak test. (F) ADCC of NMB cells opsonized with (+) or without (-) dinutuximab (dimab) by control neutrophils from healthy donors (white bars) or by neutrophils from healthy donors that were injected with G-CSF (stripped dark gray bars). N=4 control neutrophils, $n=3 \mathrm{G}-\mathrm{CSF}$ injected of two individual experiments. Statistical significances was tested with ordinary oneway ANOVA with post hoc Sidak test. ADCC, antibody-dependent cellular cytotoxicity; ANOVA, analysis of variance; G-CSF, granulocyte colony-stimulating factor; GM-CSF, granulocyte-macrophage colony-stimulating factor; MFI, mean fluorescence intensity. ${ }^{* \star} \mathrm{p}<0.01 ;{ }^{* \star \star \star} \mathrm{p}<0.0001$. 
including immunotherapy with anti-GD2 antibody dinutuximab. The treatment regimen in the USA encompasses dinutuximab administered in combination with GM-CSF and IL-2 in alternating cycles, as these have been shown to improve therapeutic efficacy. ${ }^{12}$ However, this is not the case for other countries, where GM-CSF (sargramostim) is not approved for clinical use. The limited availability of GM-CSF poses a risk of suboptimal treatment of these patients. For this reason, finding a widely available alternative stimulating cytokine that potentiates the killing of neuroblastoma cells is of high clinical relevance in areas where GM-CSF is not available. Enhancing the cytotoxic capacities of effector cells may improve dinutuximab responsiveness, which could further increase the overall survival of patients with high-risk neuroblastoma. As neutrophils are considered the main players in dinutuximab-mediated killing of neuroblastoma cells, ${ }^{8}$ we tested the capacity of neutrophils stimulated with G-CSF as opposed to GM-CSF in killing dinutuximab-opsonized GD2-positive neuroblastoma cells. For this we used both in vivo and in vitro GM-CSF or G-CSF stimulated neutrophils from either patients with neuroblastoma or healthy adult donors, and various neuroblastoma cell lines, including primary patient-derived material.

In the present study, we critically compared GM-CSF with G-CSF in the context of neutrophil ADCC of neuroblastoma cells. Previous studies showed that in vitro stimulation with GM-CSF increased the magnitude of cytotoxicity of dinutuximab-opsonized neuroblastoma cells specifically for granulocytes, while this effect was not obtained when stimulating peripheral blood mononuclear cells, emphasizing the specificity of GM-CSF on granulocytes. ${ }^{8}$ Similarly, G-CSF stimulation of neutrophils has been found to greatly enhance their capacity for ADCC in solid cancers. ${ }^{17-19}$ In this report, we found G-CSF to be as effective as GM-CSF in enhancing neutrophil ADCC of neuroblastoma cells, both after in vitro stimulation, as well as after in vivo stimulation. For the latter, we were able to use neutrophils from healthy granulocyte transfusion donors injected with G-CSF and neutrophils from patients with neuroblastoma treated with GM-CSF. Both in vivo stimulations enhanced neutrophil-mediated ADCC as opposed to unstimulated conditions, demonstrating that in vivo stimulated neutrophils can perform ADCC. We did not have access to unstimulated neutrophils of patients with neuroblastoma, but a previous report demonstrated that neutrophils of neuroblastoma patients displayed the same abilities as healthy adult neutrophils in mediating killing of neuroblastoma cells ex vivo, supporting comparison between these different cohorts. ${ }^{8}$

We found that both GM-CSF and G-CSF stimulated neutrophils induced the same effect on $\mathrm{Fc} \gamma$ receptor and integrin expression on stimulation: shedding of FcyRIIIb, and no apparent changes in expression of FcyRIIa or CD11b/CD18 integrins. We did see an increase in Fc $\gamma \mathrm{RI}$ expression after in vivo stimulation, in line with existing literature, ${ }^{37}$ and to a lesser extent also after in vitro stimulation. A previous study demonstrated that both FcrRIIa and CD11b/CD18 integrins are indispensable for neutrophil-mediated killing of antibodyopsonized solid tumor cells, ${ }^{36}$ and this is consistent with our findings in the context of dinutuximab-opsonized neuroblastoma cells; blocking CD11b/CD18 integrin function completely abolished ADCC, with Fc $\gamma$ RIIa being a dominant $\mathrm{Fc} \gamma$ receptor contributing to the process. The reason ADCG could not be fully inhibited on Fc $\gamma$ RIIa blockade is not completely understood, especially as we found that killing itself seemed to be fully antibodydependent. It might be that the concentration of Fc $\gamma$ RIIa blockade using the $\mathrm{F}(\mathrm{ab})^{\prime}{ }_{2}$ fragments was suboptimal or not complete during the 4 hours ADCC. In addition, the same might hold true for the purified IgG1 Fc tails for the saturation of Fc $\gamma$ RI.

Furthermore, neutrophils stimulated overnight with either GM-CSF or G-CSF in vitro were able to trogocytose dinutuximab-opsonized neuroblastoma cells and this was followed by tumor cell death. This is in line with previous findings where neutrophils trogocytosed trastuzumab-opsonized breast cancer cells, which led to cell rupture and death. ${ }^{36}$

Finally, we investigated the effect of G-CSF on the neuroblastoma cells. Our results showed no unfavorable effects of G-CSF during the 3-week cultures on neuroblastoma cell growth and on the susceptibility towards neutrophil-mediated ADCC in vitro, and no signs of EMT were observed. Of interest, a recent phase I/IIa clinical trial in a cohort of patients with neuroblastoma in Japan-where GM-CSF is also unavailable-assessed the tolerability, safety and feasibility of either G-CSF or macrophage colony-stimulating factor (M-CSF) during dinutuximab immunotherapy with IL-2. ${ }^{49}$ This study showed that G-CSF was welltolerated, which complements our preclinical data on the safety of G-CSF.

Although biologically very relevant, validating our findings in an in vivo mouse model would technically and methodologically be challenging. Considering syngeneic tumor mouse models, fundamental differences are observed between human and mouse neutrophil biology, reflected in the number of circulating neutrophils, function and their antitumor effects, ${ }^{50} 51$ which hamper the translatability of preclinical findings in such models. As for a xenogeneic mouse tumor model, the major obstacle is the availability of patientderived xenograft models in mice with fully functional human immune system, including neutrophils, suitable for studying dinutuximab-based immunotherapies in neuroblastoma. ${ }^{52}$ Our in vitro preclinical data on the efficacy and safety of G-CSF, together with extensive clinical experience with G-CSF in other (pediatric) indications, support direct evaluation of 
G-CSF in a clinical setting to improve immunotherapy of patients with neuroblastoma.

\section{Author affiliations}

${ }^{1}$ Department of Blood Cell Research, Sanquin Research, Amsterdam, The Netherlands

${ }^{2}$ Princess Máxima Center for Pediatric Oncology, Utrecht, The Netherlands ${ }^{3}$ Department of Experimental Immunohematology, Sanquin Research, Amsterdam, The Netherlands

${ }^{4}$ Laboratory for Immunotherapy, Sanquin Research, Amsterdam, The Netherlands ${ }^{5}$ Department of Hematopoiesis, Sanquin Research, Amsterdam, The Netherlands ${ }^{6}$ Department of Molecular Cell Biology and Immunology, Vrije Universiteit Amsterdam, Amsterdam, The Netherlands

${ }^{7}$ Department of Pediatric Immunology, Rheumatology and Infectious Diseases, Emma Children's Hospital UMC, Amsterdam, The Netherlands

\section{Twitter Waleed M Kholosy @Waleedkholosy}

Contributors PMS and DJvR designed and performed the experiments, analyzed the data and wrote the manuscript. LMJvZ, BK, PB, HO, KS, IK, AS and KL performed experiments and reviewed the manuscript. IT, MPD, WMK, JJM and GAMT provided patient material and primary patient-derived cell line 691B and reviewed the manuscript. TWK, RvB and TKvdB contributed to experimental design and data interpretation and reviewed the manuscript. HLM, GAMT and KF designed the experiments, interpreted and evaluated the data and wrote the manuscript. All authors approved the submitted version.

Funding HLM is supported by the Dutch Cancer Society (grant \#11537). IT is supported by KiKa (grant \#303) and PPOC (grant \#19-27).

\section{Competing interests None declared.}

\section{Patient consent for publication Not required.}

Ethics approval The parts of the study involving human participants were reviewed and approved by Sanquin Research Institutional Ethical Committee and Princess Maxima Center Biobank. All blood samples were obtained after informed consent and according to the Declaration of Helsinki 1964. The patients and participants provided informed consent to participate in this study.

Provenance and peer review Not commissioned; externally peer reviewed.

Data availability statement All data relevant to the study are included in the article or uploaded as supplementary information. The raw data supporting the findings of this study will be made available by the authors to any qualified researcher.

Supplemental material This content has been supplied by the author(s). It has not been vetted by BMJ Publishing Group Limited (BMJ) and may not have been peer-reviewed. Any opinions or recommendations discussed are solely those of the author(s) and are not endorsed by BMJ. BMJ disclaims all liability and responsibility arising from any reliance placed on the content. Where the content includes any translated material, BMJ does not warrant the accuracy and reliability of the translations (including but not limited to local regulations, clinical guidelines, terminology, drug names and drug dosages), and is not responsible for any error and/or omissions arising from translation and adaptation or otherwise.

Open access This is an open access article distributed in accordance with the Creative Commons Attribution Non Commercial (CC BY-NC 4.0) license, which permits others to distribute, remix, adapt, build upon this work non-commercially, and license their derivative works on different terms, provided the original work is properly cited, appropriate credit is given, any changes made indicated, and the use is non-commercial. See http://creativecommons.org/licenses/by-nc/4.0/.

\section{ORCID iD}

Paula Martinez Sanz http://orcid.org/0000-0002-3564-0505

\section{REFERENCES}

1 Whittle SB, Smith V, Doherty E, et al. Overview and recent advances in the treatment of neuroblastoma. Expert Rev Anticancer Ther 2017;17:369-86.

2 American Cancer Society 2021

3 Maris JM, Hogarty MD, Bagatell R, et al. Neuroblastoma. The Lancet 2007;369:2106-20.
4 Park JR, Bagatell R, London WB, et al. Children's Oncology Group's 2013 blueprint for research: neuroblastoma. Pediatr Blood Cancer 2013;60:985-93.

5 Smith V, Foster J. High-Risk neuroblastoma treatment review. Children 2018;5. doi:10.3390/children5090114. [Epub ahead of print: 2808 2018].

6 Ahmed M, Cheung N-KV. Engineering anti-GD2 monoclonal antibodies for cancer immunotherapy. FEBS Lett 2014;588:288-97.

7 Keyel ME, Reynolds CP. Spotlight on dinutuximab in the treatment of high-risk neuroblastoma: development and place in therapy. Biologics 2019;13:1-12.

8 Barker E, Mueller BM, Handgretinger R, et al. Effect of a chimeric anti-ganglioside GD2 antibody on cell-mediated lysis of human neuroblastoma cells. Cancer Res 1991;51:144-9.

9 Hank JA, Robinson RR, Surfus J, et al. Augmentation of antibody dependent cell mediated cytotoxicity following in vivo therapy with recombinant interleukin 2. Cancer Res 1990;50:5234-9.

10 Kushner BH, Cheung NK. GM-CSF enhances 3F8 monoclonal antibody-dependent cellular cytotoxicity against human melanoma and neuroblastoma. Blood 1989;73:1936-41.

11 Cheung IY, Hsu K, Cheung N-KV. Activation of peripheral-blood granulocytes is strongly correlated with patient outcome after immunotherapy with anti-GD2 monoclonal antibody and granulocytemacrophage colony-stimulating factor. J Clin Oncol 2012;30:426-32.

12 Yu AL, Gilman AL, Ozkaynak MF, et al. Anti-GD2 antibody with GMCSF, interleukin-2, and isotretinoin for neuroblastoma. N Engl J Med 2010;363:1324-34.

13 McGinty L, Kolesar J. Dinutuximab for maintenance therapy in pediatric neuroblastoma. Am J Health Syst Pharm 2017;74:563-7.

14 Gilman AL, Ozkaynak MF, Matthay KK, et al. Phase I study of ch14.18 with granulocyte-macrophage colony-stimulating factor and interleukin-2 in children with neuroblastoma after autologous bone marrow transplantation or stem-cell rescue: a report from the children's Oncology Group. J Clin Oncol 2009;27:85-91.

15 Ladenstein R, Pötschger U, Valteau-Couanet D, et al. Interleukin 2 with anti-GD2 antibody ch14.18/CHO (dinutuximab beta) in patients with high-risk neuroblastoma (HR-NBL1/SIOPEN): a multicentre, randomised, phase 3 trial. Lancet Oncol 2018;19:1617-29.

16 European Medicines Agency. 2020, September 12.

17 Michon J, Moutel S, Barbet J, et al. In vitro killing of neuroblastoma cells by neutrophils derived from granulocyte colony-stimulating factor-treated cancer patients using an anti-disialoganglioside/antiFc gamma RI bispecific antibody. Blood 1995;86:1124-30.

18 Treffers LW, van Houdt M, Bruggeman CW, et al. Fcyriiib restricts antibody-dependent destruction of cancer cells by human neutrophils. Front Immunol 2018;9:3124

19 Tamamori Y, Sawada T, Nishihara T, et al. GranulocyteColony stimulating factor enhances chimeric antibody ND2 dependent cytotoxicity against pancreatic cancer mediated by polymorphonuclear neutrophils. Int J Oncol 2002;21:649-54.

20 Carulli G. Effects of recombinant human granulocyte colonystimulating factor administration on neutrophil phenotype and functions. Haematologica 1997;82:606-16.

21 Gay AN, Chang S, Rutland L, et al. Granulocyte colony stimulating factor alters the phenotype of neuroblastoma cells: implications for disease-free survival of high-risk patients. $J$ Pediatr Surg 2008;43:837-42.

22 Hsu DM, Agarwal S, Benham A, et al. G-Csf receptor positive neuroblastoma subpopulations are enriched in chemotherapyresistant or relapsed tumors and are highly tumorigenic. Cancer Res 2013;73:4134-46.

23 Agarwal S, Lakoma A, Chen Z, et al. G-CSF promotes neuroblastoma tumorigenicity and metastasis via STAT3-dependent cancer stem cell activation. Cancer Res 2015;75:2566-79.

24 Green MD, Koelbl H, Baselga J, et al. A randomized double-blind multicenter phase III study of fixed-dose single-administration pegfilgrastim versus daily filgrastim in patients receiving myelosuppressive chemotherapy. Ann Oncol 2003;14:29-35.

25 Zamboni WC. Pharmacokinetics of pegfilgrastim. Pharmacotherapy 2003:23:9S-14.

26 Wendelin G, Lackner H, Schwinger W, et al. Once-per-cycle pegfilgrastim versus daily filgrastim in pediatric patients with Ewing sarcoma. J Pediatr Hematol Oncol 2005;27:449-51.

27 Borinstein SC, Pollard J, Winter L, et al. Pegfilgrastim for prevention of chemotherapy-associated neutropenia in pediatric patients with solid tumors. Pediatr Blood Cancer 2009;53:375-8.

28 Kuijpers TW, Tool AT, van der Schoot CE, et al. Membrane surface antigen expression on neutrophils: a reappraisal of the use of surface markers for neutrophil activation. Blood 1991;78:1105-11.

29 Bate-Eya LT, Ebus ME, Koster J, et al. Newly-derived neuroblastoma cell lines propagated in serum-free media recapitulate the genotype 
and phenotype of primary neuroblastoma tumours. Eur $\mathrm{J}$ Cancer 2014;50:628-37.

30 Marsman C, Jorritsma T, Ten Brinke A, et al. Flow cytometric methods for the detection of intracellular signaling proteins and transcription factors reveal heterogeneity in differentiating human $B$ cell subsets. Cells 2020;9. doi:10.3390/cells9122633. [Epub ahead of print: 0812 2020].

31 van Zogchel LMJ, Zappeij-Kannegieter L, Javadi A, et al. Specific and sensitive detection of neuroblastoma mRNA markers by multiplex RT-qPCR. Cancers 2021;13:150.

32 Stutterheim J, Gerritsen A, Zappeij-Kannegieter L, et al. PHOX2B is a novel and specific marker for minimal residual disease testing in neuroblastoma. J Clin Oncol 2008;26:5443-9.

33 Stutterheim J, Gerritsen A, Zappeij-Kannegieter L, et al. Detecting minimal residual disease in neuroblastoma: the superiority of a pane of real-time quantitative PCR markers. Clin Chem 2009;55:1316-26.

34 van Wezel EM, van Zogchel LMJ, van Wijk J, et al. Mesenchymal neuroblastoma cells are undetected by current mRNA marker panels: the development of a specific neuroblastoma mesenchymal minimal residual disease panel. JCO Precision Oncology 2019;39:1-11.

35 Metelitsa LS, Gillies SD, Super M, et al. Antidisialoganglioside/ granulocyte macrophage-colony-stimulating factor fusion protein facilitates neutrophil antibody-dependent cellular cytotoxicity and depends on FcgammaRII (CD32) and Mac-1 (CD11b/CD18) for enhanced effector cell adhesion and azurophil granule exocytosis. Blood 2002;99:4166-73.

36 Matlung HL, Babes L, Zhao XW, et al. Neutrophils kill AntibodyOpsonized cancer cells by Trogoptosis. Cell Rep 2018;23:3946-59.

37 Repp R, Valerius T, Sendler A, et al. Neutrophils express the high affinity receptor for IgG (Fc gamma RI, CD64) after in vivo application of recombinant human granulocyte colony-stimulating factor. Blood 1991;78:885-9.

38 Treffers LW, Hiemstra IH, Kuijpers TW, et al. Neutrophils in cancer. Immunol Rev 2016;273:312-28.

39 Peipp M, Lammerts van Bueren JJ, Schneider-Merck T, et al. Antibody fucosylation differentially impacts cytotoxicity mediated by NK and PMN effector cells. Blood 2008;112:2390-9.
40 Bouti P, Zhao XW, Verkuijlen PJJH, et al. Kindlin3-Dependent CD11b/CD18-Integrin activation is required for potentiation of neutrophil cytotoxicity by CD47-SIRP $\alpha$ checkpoint disruption. Cancer Immunol Res 2021:9:147-55.

41 Tabiasco J, Espinosa E, Hudrisier D, et al. Active trans-synaptic capture of membrane fragments by natural killer cells. Eur $J$ Immunol 2002;32:1502-8.

42 Velmurugan R, Challa DK, Ram S, et al. Macrophage-Mediated trogocytosis leads to death of Antibody-Opsonized tumor cells. Mol Cancer Ther 2016;15:1879-89.

43 Ladenstein R, Valteau-Couanet D, Brock P, et al. Randomized trial of prophylactic granulocyte colony-stimulating factor during rapid COJEC induction in pediatric patients with high-risk neuroblastoma: the European HR-NBL1/SIOPEN study. J Clin Oncol 2010;28:3516-24.

44 Maris JM, Healy J, Park J, et al. G-CSF is a cancer stem cell-specific growth Factor-Letter. Cancer Res 2015;75:3991.

45 Schneider A, Krüger C, Steigleder T, et al. The hematopoietic factor G-CSF is a neuronal ligand that counteracts programmed cell death and drives neurogenesis. J Clin Invest 2005;115:2083-98.

46 Thiery JP. Epithelial-Mesenchymal transitions in tumour progression. Nat Rev Cancer 2002;2:442-54.

47 Yang J, Weinberg RA. Epithelial-Mesenchymal transition: at the crossroads of development and tumor metastasis. Dev Cell 2008;14:818-29.

48 van Groningen T, Koster J, Valentijn LJ, et al. Neuroblastoma is composed of two super-enhancer-associated differentiation states. Nat Genet 2017;49:1261-6.

49 Hara J, Nitani C, Kawamoto H, et al. A phase I/lla study of Antidisialoganglioside antibody Dinutuximab in Japanese patients with neuroblastoma. J Pediatr Hematol Oncol 2021;43:e358-64.

50 Eruslanov EB, Singhal S, Albelda SM. Mouse versus human neutrophils in cancer: a major knowledge gap. Trends Cancer 2017;3:149-60.

51 Bruhns P, Jönsson F. Mouse and human FcR effector functions. Immunol Rev 2015;268:25-51.

52 Braekeveldt N, Bexell D. Patient-Derived xenografts as preclinical neuroblastoma models. Cell Tissue Res 2018;372:233-43. 